\title{
Supresión de la lactancia materna exclusiva en menores de seis meses con alergia a la proteína de leche de vaca ${ }^{1}$
}

\author{
Marilyn Barrantes Rojas ${ }^{2}$ \\ Maricela Ruiz Acuña ${ }^{3}$
}

Institución: Universidad de Costa Rica, Maestría en Enfermería Pediátrica

\section{RESUMEN}

La alergia a la proteína de leche de vaca es considerada actualmente una epidemia dentro de la categoría de enfermedades no contagiosas, causa principal de alergia en menores de un año, cuya incidencia aumenta alrededor del mundo, por ello muchos de los niños diagnosticados son privados de una lactancia materna exclusiva a pesar de que sigue siendo protectora frente a los síntomas, razón por la cual es de vital importancia que los profesionales de la salud cuenten con la información más actualizada y disponible para continuar protegiendo y promoviendo la lactancia materna en estos niños de manera que se fortalezca su sistema inmunológico y se vigile la evolución del lactante sin poner en riesgo su adecuado crecimiento y desarrollo, así como la continuidad de la lactancia materna. El objetivo de esta revisión breve es analizar la evidencia científica disponible respecto de la supresión de la lactancia materna exclusiva en niños menores de seis meses con sospecha de alergia a la proteína de la leche de vaca como una estrategia para mejorar los síntomas. Se utilizó como metodología la práctica clínica basada en la evidencia: inicialmente se planteó una pregunta clínica de tipo pronóstico y objetivos acordes a esta, con base en los que se identificó un conjunto de descriptores a partir de los que se realizó la búsqueda de evidencia científica en diferentes bases de datos.

Palabras claves: alergia-a-proteína-de-leche-de-vaca, lactancia-materna-exclusiva, hipersensibilidad-enlactantes.

\footnotetext{
${ }^{1}$ Fecha de recepción: 5 de diciembre 2017

Fecha de aceptación: 23 de marzo del 2018

${ }^{2}$ Enfermera, estudiante de maestría en Enfermería Pediátrica con énfasis en Neonatología. Universidad de Costa Rica. Consultora Internacional de Lactancia Materna, Lactamar. Costa Rica. Correo electrónico: lactamarcr@gmail.com, marybr9@@hotmail.com ${ }^{3}$ Enfermera, estudiante de maestría en Enfermería Pediátrica con énfasis en Neonatología. Universidad de Costa Rica. Consultora Internacional de Lactancia Materna. Hospital CIMA San José. Costa Rica. Correo electrónico: ce-hunny@hotmail.com
} 


\title{
Exclusion of exclusive breastfeeding in children under six months with allergy to cow's milk $\operatorname{protein}^{1}$
}

Institution: University of Costa Rica, Postgraduate Pediatric Nursing

\author{
Marilyn Barrantes Rojas ${ }^{2}$ \\ Maricela Ruiz Acuña ${ }^{3}$
}

\begin{abstract}
The allergy to cow's milk protein is currently considered an epidemic within the category of non-communicable diseases, is the main cause of allergy in children under one year and continues to increase its incidence around the world, many of the children diagnosed are deprived of exclusive breastfeeding despite the fact that it remains protective against symptoms, which is why it is vitally important that health professionals have the most up-todate and available information to continue protecting and promoting breastfeeding in these children in a way that strengthens their immune system and monitors the evolution of the infant without jeopardizing the continuity of breastfeeding and its adequate growth and development. The objective of this brief review is to analyze the available scientific evidence regarding the suppression of exclusive breastfeeding in children under 6 months of age with suspected cow's milk protein allergy as a strategy to improve symptoms. The clinical practice based on evidence was used as a methodology, initially a clinical question of prognostic type was raised and the objectives according to this were raised, based on which a set of descriptors was identified from which it was made the search for scientific evidence in different scientific databases.
\end{abstract}

Keywords: allergy-to-cow-milk-protein, exclusive-maternal-lactation, hypersensitivity-in-infants

\footnotetext{
${ }^{1}$ Date of receipt: December 5, 2017

Date of acceptance: March 23, 2018

${ }^{2}$ Nurse, Master's student in Pediatric Nursing with an emphasis in Neonatology. University of Costa Rica. International Breastfeeding Consultant, Lactamar. Costa Rica. E-mail: lactamarcr@gmail.com, marybr 9@,hotmail.com

${ }^{3}$ Nurse, Master's student in Pediatric Nursing with an emphasis in Neonatology. University of Costa Rica. International Breastfeeding Consultant. CIMA San José Hospital. Costa Rica. E-mail: ce-hunny@,hotmail.com
} 


\title{
Exclusão da amamentação exclusiva em crianças menores de seis meses com alergia à proteína do leite de vaca ${ }^{1}$
}

\author{
Marilyn Barrantes Rojas ${ }^{2}$ \\ Maricela Ruiz Acuña ${ }^{3}$
}

Instituição: Universidade da Costa Rica, Enfermagem pediátrica de pós-graduação

\section{RESUMO}

A alergia à proteína do leite de vaca é atualmente considerada uma epidemia na categoria de doenças não transmissíveis, é a principal causa de alergia em crianças menores de um ano e continua a aumentar sua incidência em todo o mundo, muitas das crianças diagnosticadas são privado de aleitamento exclusivo, apesar de permanecer protetor contra os sintomas, razão pela qual é de vital importância que os profissionais de saúde tenham a informação mais atualizada e disponível para continuar a proteger e promover a amamentação em essas crianças de uma forma que fortalece seu sistema imunológico e monitora a evolução da criança sem comprometer a continuidade da amamentação e seu próprio crescimento e desenvolvimento. O objetivo desta breve revisão é analisar a evidência científica disponível sobre a supressão da amamentação exclusiva em crianças com menos de 6 meses de idade com suspeita de alergia a proteínas de leite de vaca como estratégia para melhorar os sintomas. A Prática Clínica baseada na evidência foi utilizada como metodologia, inicialmente foi levantada uma questão clínica de tipo prognóstico e os objetivos de acordo com isso foram levantados, com base nos quais um conjunto de descritores foi identificado a partir do qual foi feito a busca de evidências científicas em diferentes bancos de dados científicos.

Palavras-chave: exclusão-lactação-materna, hipersensibilidade-em-lactentes, proteína-de-alergia-a-leite-de-vaca

\footnotetext{
${ }^{1}$ Data de recepção: 5 de dezembro de 2017

Data de aceitação: 23 de março de 2018

${ }^{2}$ Enfermeira, aluna de mestrado em enfermagem pediátrica com ênfase em neonatologia. Universidade da Costa Rica. Consultora Internacional de Aleitamento Materno, Lactamar. Costa Rica. Correio eletrônico: lactamarcr@gmail.com, marybr 9@,hotmail.com ${ }^{3}$ Enfermeira, aluna de mestrado em enfermagem pediátrica com ênfase em neonatologia. Universidade da Costa Rica. Consultor Internacional de Aleitamento Materno. Hospital CIMA San José. Costa Rica. Correio eletrônico: ce-hunny@hotmail.com
} 


\section{Revista Electrónica Entermeria actual en costa Rica}

\section{INTRODUCCIÓN}

La alergia a la proteína de leche de vaca (APLV) es un trastorno frecuente en la infancia y representa "la patología por alergia alimentaria más común en el niño pequeño"17: se trata de una reacción de hipersensibilidad determinada por mecanismos inmunológicos específicos, considerada un problema de salud que pone en riesgo el desarrollo adecuado de la tolerancia oral en el infante, ${ }^{18}$ así como su adecuado crecimiento y desarrollo.

Al respecto, en 1976, en Costa Rica, Monge ${ }^{1}$ realizó un estudio descriptivo de 32 casos clínicos de alergia a la proteína de leche de vaca que se presentaron en niños en el Hospital Nacional de Niños con el fin de evaluar los factores de riesgo asociados, la sintomatología y el inicio del cuadro clínico: se encontró que los síntomas se presentaban en niños que no han sido amamantados al seno materno o que han sido amamantados por periodos de seis meses a un año, los cuales comienzan a aparecer pocas semanas o meses después de recibir leche de vaca en su alimentación. Concluye que la alergia a la leche de vaca es una entidad patológica que se ha detectado desde que las madres han abandonado la lactancia al pecho materno.

Para el año 1983, Hanson y Porras ${ }^{2}$ hicieron un estudio titulado Aspectos inmunológicos de la leche materna, cuyo objetivo fue elaborar una revisión de tipo descriptiva sobre los principales componentes de la leche materna que previenen las infecciones y alergias en los recién nacidos para ser considerada como una importante fuente de protección del lactante. En cuanto a lo mencionado, mencionan que la leche materna contiene también anticuerpos contra proteínas alimentarias, incluso, contra la proteína de la leche de vaca, sin olvidar el factor epidérmico de crecimiento que puede influir en la maduración del epitelio de la mucosa intestinal y en su capacidad de manejar alergenos potenciales. Concluyen que no está claro si estos anticuerpos pueden disminuir la exposición de la mucosa intestinal a la proteína durante la ablactación si se continúa la lactancia materna, además de que son necesarios más estudios al respecto.

Por otro lado, en el año 1997, Chavarria ${ }^{3}$, también en Costa Rica, realizó un estudio descriptivo en el Hospital Nacional de Niños en el que se examinó las respuestas dadas a un formulario por los padres o encargados de 60 niños/niñas referidos a la consulta de alergología para su valoración y que tuviera un pariente cercano alérgico. El formulario interrogaba sobre duración de la lactancia materna, inicio de la ingesta de leche de vaca, de huevo y de tomate. Se encontró que a más de la mitad de los niños se les había suspendido la lactancia materna al cumplir seis meses y que en el primer año, más de la mitad de los niños recibieron leche de vaca y huevo. Concluyeron que en la población costarricense con riesgo de padecer enfermedad alérgica, no existe conciencia de la importancia de la lactancia materna por tiempo prolongado, ni de la utilidad de evitar alimentos altamente alergizantes en los primeros meses de la vida.

En el año 2009, se publica el informe final de la Gerencia Médica de la Caja Costarricense del Seguro Social $^{4}$ (CCSS) sobre el Protocolo para uso institucional de fórmula enteral de nutrientes complejos con proteína de soya para niños con diagnóstico de alergia a la proteína de leche de vaca, cuyo objetivo fue protocolizar la atención a estos niños que reciben atención en centros públicos: al respecto, establece que la fórmula con proteína de soya no se debe utilizar en infantes menores de seis meses de edad y que, en menores de un año -especialmente los menores de seis meses-, se debe evitar la exposición a fórmulas modificadas con proteína de leche de vaca o leche entera de vaca, por el riesgo de alergia, principalmente si tienen factores de 


\section{Revista Electrónica Entermeria actual en costa Rica}

riesgo. Por otra parte, mencionan que los niños alimentados exclusivamente con leche materna que presentan síntomas de alergia alimentaria, deben ser valorados por un especialista en pediatría. Al efecto, se debe proceder y continuar la ingesta de leche materna con restricción de proteínas de leche de vaca en la dieta materna y brindar control en la clínica de lactancia. Se recalca y concluye que la leche materna constituye la fuente óptima para la nutrición de lactantes e infantes pequeños; por lo tanto, los esfuerzos institucionales deben dirigirse a favorecer el uso de este recurso materno como prioridad.

En el año 2010, Celada y Celada ${ }^{5}$ publicaron el artículo Antecedentes familiares de alergia en recién nacidos atendidos durante el primer semestre del 2009 en el Hospital San Juan de Dios, en Costa Rica, un estudio observacional en el que, a través de un interrogatorio, se determina la presencia de antecedentes alérgicos en las madres, los padres y los hermanos de 168 recién nacidos consecutivos durante el primer trimestre del año 2009 en ese hospital. El objetivo fue identificar los factores de riesgo para desarrollar alergias, respecto de lo que se encontró que el $23 \%$ de las madres ya han introducido fórmulas en la alimentación de sus hijos para los 15 días de edad y que para los tres meses, más de un tercio la han recibido, lo cual se considera un factor de riesgo importante. Concluye que para evitar la exposición temprana a antígenos alimentarios como la proteína de leche de vaca se debe brindar la lactancia materna exclusiva por al menos los cuatro primeros meses de vida.

En esa misma línea, en el 2004, en México, Carvajal ${ }^{6}$ realizó un estudio longitudinal, Lactancia materna en el primer semestre y la salud de los niños en el primer año de vida, en el que estudió a 170 infantes -85 lactados a pecho por menos de seis meses- a los cuales llamó grupo expuesto, y 85 con que recibieron lactancia materna exclusiva mínimo por seis meses o más, a los cuales llamó grupo no expuesto: el objetivo fue la protección que brinda la leche materna a los recién nacidos, en temas de patologías como infección, alergia y demás, de cuyos resultados obtuvo que en los infantes expuestos a la lactancia materna en menor tiempo aumentó la incidencia de dichas patologías, comparados con quienes la recibieron por más tiempo.

Por otro lado, en Chile, en el año 2011, Rossel $^{7}$ desarrolló una revisión sobre la alergia alimentaria en la infancia, cuyo objetivo era evaluar las prácticas dietéticas seguidas en la primera infancia por una población con riesgo genético de sensibilización: se revisó y discutió aspectos relacionados con la definición, epidemiología, principales alergenos, tipos de reacciones inmunes asociadas a los cuadros alérgicos, diagnóstico y tratamiento de las alergias alimentarias: entre las conclusiones ese obtuvo que la alergia alimentaria se ha transformado en un cuadro de alta prevalencia, cuyo tratamiento consiste en eliminar los agentes causales de la dieta, la cual debe mantenerse equilibrada tanto para el infante, como para la madre, en caso de que sea amamantado.

En ese mismo año, en Francia, Denis, Loras-Duclaux y Lachaux ${ }^{8}$ publicaron Sensibilisation et allergie aux proteines du lait de vache chez l'enfant allaite, cuyo objetivo fue describir la importancia del rol de la leche materna en el mejoramiento de los síntomas de los lantantes: al respecto, concluyeron que la prohibición de PLV materna es la única solución compatible con la lactancia en estos casos y que el pronóstico del APLV infantil es a menudo favorable con la adquisición de una tolerancia en el infante antes de sus tres o cuatro años en casi el $80 \%$ de los casos. Se menciona, además, que la leche materna sigue siendo la mejor alternativa para los lactantes diagnosticados APLV ya que logra satisfacer de forma completa las necesidades nutricionales e inmunológicas de lactante.

En el año 2012, De Greef et al. ${ }^{9}$ publicaron una revisión sistemática en Bélgica sobre el diagnóstico y manejo de los niños con alergia a la proteína de leche de vaca, en la que buscaron la mejor evidencia disponible 


\section{Revista Electrónica Entermeria actual en costa Rica}

que guiara el manejo: mencionan que existen diferencias en el tratamiento dependiendo del tipo de alimentación que recibe el infante previo a su diagnóstico ya que difiere si este es exclusivamente amamantado o alimentado con fórmula y que, debido a los múltiples beneficios que otorga la lactancia materna, se recomienda continuar amamantando con dieta de exclusión materna en casos confirmados de APLV y, solo en caso de que la madre desee destetar, se recomienda el uso de una fórmula especial, mientras que, en el caso de los bebés que son alimentados con fórmula, el uso de otra a base de aminoácidos esenciales es la línea de tratamiento. Se destaca el papel preventivo que tiene la lactancia materna exclusiva para el desarrollo de alergias y se recomienda continuar amamantando frente al diagnóstico de APLV, ya que representa la mejor opción, además de que es más económica, para el mejoramiento de los síntomas.

El Ministerio de Salud del Gobierno de Chile publicó en el año 2013 la Guía clínica basada en evidencia para el manejo de niños menores de dos años con alergia a la proteína de leche de vaca ${ }^{10}$ en la que se destaca la recomendación de proteger la lactancia materna exclusiva en menores de seis meses de edad y considerar dieta de eliminación de PLV materna en quienes son alimentados con lactancia materna, ambos con grado de recomendación (C). De igual forma, se recomienda confirmar APLV mediante dieta de eliminación materna y contraprueba en niños con sospecha de APLV leve a moderada así como indicar fórmula láctea extensamente hidrolizada o fórmula elemental en menores con APLV que son alimentados con fórmula, ambas con grado de recomendación (B).

En el año 2015, en México, Toro et al. ${ }^{11}$ publicaron un estudio comparativo, retrospectivo, transversal, observacional, en el que incluyeron a 101 niños menores de un año con diagnóstico de APLV con el fin de analizar factores perinatales de riesgo para el desarrollo de alergia a la proteína de leche de vaca, ya que encontraron que los niños con este diagnóstico tenían una duración menor en meses de lactancia materna exclusiva, lo cual se considera un factor de riesgo. Se recomienda fomentar la lactancia materna con la finalidad de disminuir la APLV, cuyo costo de tratamiento es elevado, dada la necesidad de empleo de fórmulas especiales.

En el año 2015, Montero ${ }^{12}$ realizó un estudio descriptivo, retrospectivo sobre la alergia a la proteína de la leche de vaca, titulado Estudio descriptivo intervencionista sobre la alergia a la proteina de la leche de vaca IGE mediada en niños del área de salud de Mérida, cuyo objetivo fue estudiar la incidencia de la alergia a la proteína de la leche de vaca y el tratamiento que se le puede brindar. El estudio fue divido en dos fases, en las que se incluyen menores - estudiados de manera individual- con sospecha de presentar alergia a la proteína de la leche de vaca, la cual explica cómo fue abordada su intervención diagnóstica y después el tratamiento, con lo que concluye que es importante realizar un correcto diagnóstico de APLV en lactantes, para evitar el sobrediagnóstico, por tanto, se debe realizar un estudio con el alergólogo antes de instaurar un sustitución en la dieta, en muchas ocasiones innecesaria.

También en el año 2015, en Australia, se publicó Milk Matters; Infant Feeding and Inmune Disorder ${ }^{13}$ de Maureen Mitchin, educadora en salud, elaborado a lo largo de 30 años de experiencia clínica, dando apoyo a familias con niños APLV. Se investiga sobre la leche materna respecto de la función inmune: los efectos de los sustitutos de la leche materna, el papel de la alimentación en el comportamiento infantil (especialmente los cólicos), y la necesidad de apoyo en ese ámbito, se ha convertido en la fuente más completa para la investigación sobre la alimentación infantil, así como lo relacionado con los efectos sobre el sistema inmune y funcionamiento que existe en la actualidad. Se argumenta que el daño inmune ha sido adquirido a través de la nutrición infantil 


\section{Revista Electrónica Entermeria actual en costa Rica}

temprana, y se transmite intergeneracionalmente. Además, se documenta el experimento de la vida real de las fórmulas infantiles, y sus consecuencias conocidas y emergentes para la salud temprana y para toda la vida; finalmente, se establece una relación entre la ciencia y la historia con los problemas infantiles cotidianos, se brinda consejos prácticos sobre las controversias actuales y previene o resuelve la angustia relacionada con la dieta en infantes, además de que se argumenta a favor de un vínculo entre la alimentación artificial y una serie de problemas inmunes y de comportamiento en los lactantes y en menores, sobre todo respecto de la APLV.

En el año 2016, Carmargo et al. ${ }^{14}$ publicaron un estudio de cohorte hecho en Brazil que incluye recién nacidos con diagnóstico de proctocolitis alérgica por proteína de leche de vaca seguidos en el Instituto de Gastroenterología Pediátrica de São Paulo. El objetivo fue evaluar el estado nutricional de los bebés de acuerdo con la intervención terapéutica nutricional de elección, lactancia materna exclusiva con dieta materna de eliminación de alergenos y utilización de fórmulas extensamente hidrolizadas sin encontrar diferencias en ambos grupos, cuyo resultado fue la remisión total de los síntomas clínicos: entre las conclusiones se destaca el papel beneficioso y altamente efectivo de la lactancia materna exclusiva sin alimentos alergenos en el proceso de mantenimiento nutricional de estos infantes y remisión de la sintomatología, por lo que se recomienda fomentar esta práctica para favorecer el estado nutricional, así como el vínculo afectivo, con el fín de evitar la introducción de fórmulas hipoalergénicas que pueden representar mayores costos en salud.

En el año 2016, Errázuriz et al. ${ }^{15}$ publicaron Características clínicas y manejo de lactantes menores de u año con sospecha de alergia a proteína de leche de vaca, un estudio retrospectivo ,cuyo objetivo fue describir características demográficas, clínicas y manejo de lactantes $<1$ año con sospecha de APLV. Se incluyó 106 lactantes, $51 \%$ varones, $80 \%$ recién nacidos de término, $74 \%$ con al menos un progenitor atópico, $34 \%$ con alguno de los padres o algún hermano con alergia alimentaria. La mediana de edad al inicio fue 1,5 meses. Se encontró que el 15\% recibió fórmula desde el periodo neonatal y el 50\% antes del tercer mes, lo cual coincide con el momento en que iniciaron sus síntomas la mayoría de los pacientes. El 35\% pudo mantener la lactancia materna exclusiva, con dieta de restricción de la madre, mayoritariamente suspensión de lácteos y soja, eventualmente. En esta serie se evidenció que la respuesta en estos casos fue satisfactoria. Cuando el paciente no recibió lactancia materna por distintas razones se indicó fórmula extensamente hidrolizada o basada en aminoácidos (24\% y $26 \%$ respectivamente). La respuesta al cambio de dieta en función de la apreciación de los padres fue en un $94 \%$ positiva, grupo que incluye la desaparición total y parcial de síntomas. Se concluye que en pacientes alimentados al pecho es necesario mantener la lactancia materna e indicar dieta a la madre.

Nyankovskyy et al. ${ }^{16}$ hicieron un estudio multicéntrico prospectivo en Ucrania en el año 2016, el cual incluyó a 30 menores de un año alimentados con fórmula exclusiva y con diagnóstico de alergia severa a la proteína de leche de vaca, con el fin de comprobar la eficacia clínica de la fórmula de aminoácidos en esos bebés para la reducción de los síntomas. No participaron infantes que fueran amamantados; se identificó como factor de riesgo en el inicio de los síntomas el uso de fórmulas lácteas. El estudio concluye que la utilización de fórmula a base de aminoácidos demostró ser efectiva para reducir los síntomas -tanto gastrointestinales como dermatológicos- de alergia severa.

La lactancia materna ha demostrado ser protectora para el sistema inmunológico en desarrollo, ya que tiene un papel altamente evolucionado en la programación y funcionamiento del sistema inmune del neonato inmaduro, en cuanto a que ayuda en la estructuración y fortalecimiento de una microbioma intestinal que 
funcionará como barrera protectora y defensora del lactante, la cual mantiene comunicación directa con la mama a través del sistema enteromamario ${ }^{19-20}$. Lo anterior es de gran relevancia para el lactante, además de que sirve a la vez como incentivo para la protección y promoción de la lactancia materna desde el nacimiento, ya que se ha demostrado ampliamente que la leche materna es un puente único entre el útero y el mundo externo, la cual ayuda en la modulación de la respuesta del infante al medioambiente, para programar no solo un crecimiento y desarrollo óptimos, sino también respuestas adecuadas a los desafíos que enfrente. ${ }^{20}$

En torno al tema, se estima que de 1.3 a 1.45 millones de muertes en 42 países de alta mortalidad podrían ser prevenidas aumentando los niveles de lactancia ${ }^{19}$, motivo por el que es importante realizar un diagnóstico, ya que la respuesta terapéutica de eliminar la leche materna como alimento, no puede ser abordada de una forma sencilla.

Las enfermedades alérgicas tienen una prevalencia creciente a nivel nacional y a mundial, que impactan significativamente la salud pública ${ }^{21}$, debido a que muchos de los lactantes menores de seis meses con diagnóstico o sospecha de alergia a la proteína de leche de vaca terminan utilizando una fórmula infantil exclusiva, ya que dentro de la práctica clínica no se ha establecido aún con claridad la importancia de la promoción y protección de la lactancia materna en estos casos que, por lo general, privan al infante de este beneficio y le refuerzan a la madre la causalidad directa de la exposición por dieta materna y la mejoría inmediata con la sustitución de esta con fórmulas especialmente diseñadas.

De forma ampliamente extendida en la investigación, se recomienda la lactancia exclusiva hasta los seis meses, por su efecto prebiótico favorable sobre la microbiona intestinal y el desarrollo inmunológico del lactante $\mathrm{e}^{2021-10-11-12}$, práctica favorable aún frente al diagnóstico de alergia, ya que se ha demostrado un efecto protector ${ }^{8-10-11-12}$ máxime que, como bien lo indica la Comisión Nacional de Alergias de Argentina, "la lactancia materna es la dieta de elección más allá de su influencia sobre el desarrollo de alergias" "21, aun cuando siempre se ha culpado la leche materna, sin considerar que la inclusión de enzimas bovinas en la dieta prenatal y en la de los lactantes, crea una sensibilización desde el útero con el consecuente paso de anticuerpos placentarios que repercuten en el lactante.

La importancia de la temática va más allá de la complejidad de la alergia, debido a que la práctica de sustitución de la lactancia natural por la fórmula láctea transgrede la salud de los infantes, lo cual genera cambios intergeneracionales en la microbiana que altera los procesos de crecimiento y desarrollo adecuados, sin olvidar que cada vez aumentan más los casos de alergia, cáncer infantil y demás patologías.

Dado lo anterior, la presente revisión breve tiene como objetivo analizar la mejor evidencia científica disponible respecto de la supresión de la lactancia materna exclusiva en menores de seis meses con sospecha de alergia a la proteína de la leche de vaca, como una estrategia para mejorar los síntomas.

\section{MÉTODO}

Se aplicará la metodología de práctica clínica basada en la evidencia la cual establece cinco pasos: 1 . Establecimiento de una pregunta clínica 2. Búsqueda de la mejor evidencia científica 3. Análisis crítico de la 
mejor evidencia encontrada 4. Transferencia de la evidencia científica y 5. Evaluación de la transferencia de la evidencia científica.

\section{Pregunta clínica}

La pregunta de revisión es la siguiente: ¿En niños menores de seis meses con sospecha de alergia a la proteína de leche de vaca, es la supresión de la lactancia materna exclusiva una práctica que ayuda a la mejoría de los síntomas? Lo anterior se desglosa en el siguiente acrónimo PICO:

\begin{tabular}{|l|l|c|c|}
\hline \multicolumn{1}{|c|}{ Población } & \multicolumn{1}{|c|}{ Intervención } & Comparación & Resultado (Outcome) \\
\hline $\begin{array}{l}\text { Niños menores de seis } \\
\text { meses con sospecha de }\end{array}$ & $\begin{array}{l}\text { Supresión de la lactancia } \\
\text { materna exclusiva }\end{array}$ & - & Mejora la sintomatología \\
alergia a la proteína de la & & & \\
lecha de vaca & & & \\
\hline
\end{tabular}

El tipo de pregunta se clasifica como una pregunta de "pronóstico" que utiliza estudios de cohorte longitudinal, para responderla.

\section{Criterios de inclusión}

Población: se considerará estudios que incluyan niños menores de seis meses que hayan sido diagnosticados con sospecha de alergia a la proteína de la lecha de vaca, independientemente de morbilidad asociada, sexo, días de nacido y edad gestacional.

Intervención: se incluirá estudios que reporten la supresión de la lactancia materna exclusiva.

Outcome (resultado): se considerará estudios que establezcan los resultados relacionados con la supresión de la lactancia materna exclusiva y sus efectos sobre la sintomatología que presentan los infantes.

\section{Tipos de estudio}

Se incluirá los estudios publicados no experimentales; los diseños incluyen estudios de cohorte longitudinal, cuya publicación esté en idioma español, inglés, portugués y francés.

\section{Estrategia de búsqueda}

La estrategia de búsqueda tendrá como objetivo encontrar estudios científicos publicados, la cual seguirá tres pasos: inicialmente se llevará a cabo una búsqueda limitada en MedLine, Google Scholar, EBSCO y Cochrane Plus por afinidad con el título de la investigación, además de considerar que el resumen de la investigación contenga -claramente expuestos- los elementos de relevancia en este apartado, como lo son el 


\section{Revista Electrónica Enfermeria Actual en costa Rica}

objetivo de la investigación, la metodología, así como resultados y conclusiones principales. Luego, se realizará una segunda búsqueda utilizando los descriptores planteados en esta revisión en las bases de datos PubMed, Cochrane, Science Direct, Scielo y Clinical Key. En tercer lugar, se buscará estudios adicionales en la lista de referencias de todos los informes y artículos identificados. Se incluirá toda la información disponible entre el 2013 y el 2017.

\section{Descriptores de búsqueda}

Para la búsqueda de información se utilizará los descriptores, en español y en inglés, expuestos en la tabla 1, utilizando AND, OR, NOT.

Tabla 1. Descriptores para la búsqueda de información

\begin{tabular}{l|l|l|l}
\hline \multicolumn{1}{|c|}{ Español } & \multicolumn{1}{c}{ Inglés } & \multicolumn{1}{c}{ Portugués } & \multicolumn{1}{c}{ Francés } \\
\hline $\begin{array}{l}\text { Alergia a la proteína de leche } \\
\text { de vaca }\end{array}$ & Allergy to cow's milk protein & $\begin{array}{l}\text { Alergia à proteína do leite de } \\
\text { vaca }\end{array}$ & $\begin{array}{l}\text { Allergie aux protéines du lait } \\
\text { de vache }\end{array}$ \\
\hline Lactancia materna exclusiva & Exclusive breastfeeding & Aleitamento materno exclusivo & Allaitement maternel exclusif \\
\hline Alergia a la leche & Milk allergy & Alergia ao leite & Allergie au lait \\
\hline Hipersensibilidad a la leche & Hypersensitivity to milk & Hipersensibilidade ao leite & Hypersensibilité au lait \\
\hline Alergia a la caseína & Casein allergy & Alergia a caseína & Allergie à la caséine \\
\hline
\end{tabular}

Fuente: elaboración propia

\section{Selección de los estudios}

Luego de la búsqueda de información, todas las citas identificadas se recopilará y se cargará en el software bibliográfico Mendeley para eliminar los duplicados. Se examinará los títulos y los resúmenes para evaluarlos según los criterios de inclusión para la revisión. Los estudios que cumplan con los criterios de inclusión se recuperará en su totalidad y se importará sus detalles en una matriz de datos de Excel. El texto completo de los estudios seleccionados se recuperará y evaluará en detalle en relación con los criterios de inclusión. Se excluirá los estudios de texto completo que no cumplan con los criterios de inclusión y se proporcionará los motivos de la exclusión en un apéndice del informe de la revisión final.

Los resultados de la búsqueda se anotará en su totalidad en el informe final y se presentará en un diagrama de flujo PRISMA 2009 en su versión en español.

Cualquier desacuerdo que surja entre los investigadores se resolverá mediante una discusión conjunta.

\section{Extracción de los datos}

Se extraerá los datos de los artículos incluidos en la revisión de forma manual, que aportarán detalles específicos, como año, autor, título, tipo y métodos del estudio, participantes, tipo de intervención, selección de la 
muestra, resultado principal y secundario de importancia para la pregunta clínica planteada y los objetivos específicos.

Cualquier desacuerdo entre los investigadores se resolverá mediante una discusión conjunta. Se contactará a los autores de los documentos para solicitar datos faltantes o adicionales cuando sea necesario.

\section{Evaluación de la calidad metodológica}

Para evaluar la calidad metodológica de la documentación recuperada, se desarrollará un análisis crítico de la evidencia según lo recomiendan Rojas, Cubero y Leiva ${ }^{16}$.

La calidad de la información y su pertinencia para dar respuesta a la pregunta planteada de la investigación se hará a través de la plataforma de fichas de lectura crítica 2.0 (FLC 2.0) que permite analizar la calidad o validez de los artículos científicos según el nivel bajo, medio o alto. Por otro lado, también se puede utilizar el Critical Appraisal Skills Programme español (CASPe) que proporciona una serie de plantillas según el tipo de estudio que se evalúe para ayudar al investigador a emitir un criterio respecto del documento evaluado en términos de calidad y validez. El grado de recomendación será asignado según el Centre for Evidence-Based Medicine, Oxford (OCEBM)

\section{Síntesis de datos}

Los resultados de esta revisión breve serán sintetizados y presentados en forma de tabla de evidencia y resumen narrativo de acuerdo con los objetivos específicos para su análisis posterior.

\section{Conflicto de intereses}

La autora declara que no tiene conflictos de tipo personal, comercial, académico, político o financiero.

\section{Agradecimiento}

Agradecemos el apoyo de Marta Trejos y Maureen Mintchin por el préstamo de la literatura y equipo del libro "Milk Matters".

\section{REFERENCIAS BIBLIOGRÁFICAS}

1. Monge J. Alergia a la leche de vaca. Revista Médica de Costa Rica. 1976; XLIII(455):65·71.

2. Hanson L, Porras O. Aspectos Inmunológicos de la Leche Materna. Rev Méd Hosp Nac Niños Costa Rica. 1983;1(18):69-86.

3. Chavarría J. Prácticas dietéticas utilizadas en niños costarricenses con riesgo de padecer enfermedad alérgica. Acta Pediátrica Costarricense. 1997;1(11):23-25. 


\section{Revista Electrónica Enfermeria Actual en costa Rica}

\section{CCSS. PROTOCOLO PARA USO INSTITUCIONAL DE FÓRMULA ENTERAL DE NUTRIENTES COMPLEJOS CON PROTEÍNA DE SOYA. San José, Costa Rica.: Gerencia Médica; 2008 p. 7-8p.}

5.Celada R, Celada V. Antecedentes de Alergia en Recién Nacidos atendidos durante el primer trimestre del 2009 en el Hospital San Juan de Dios. Acta Pediátrica Costarricense. 2010;1(22):28-33.

6. Carvajal, C. Lactancia materna en el primer semestre y la salud de los niños en el primer año de vida. REV. MED.MEX. PEDIATRIA. 2004;7(5).

7. Rossel M. Alergia Alimentaria en la Infancia. REV. MED. CLIN. CONDES. 2011; 22(2): 184-189.

8. Denis M, Loras-Duclaux I, Lachauxa A. Sensibilisation et allergie aux prote' ines du lait de vache chez l'enfant allait. Archives de Pediatrie. 2012;(19):305-312.

9. De Greef E, Hauser B, Devreker T, Veereman-Wauters G, Vandenplas Y. Diagnosis and management of cow's milk protein allergy in infants. World J Pediatr. 2012; 8:19-24. http://dx.doi.org/10.1007/s12519-012-0332-X

10. Ministerio de Salud, Gobierno de Chile. Guía Clínica para el manejo de la alergia a la proteína de leche de vaca. Chile: Subsecretaría de Salud Pública; 2012 p. 15-19 p.

11. Miquel, I. y Arancibia, ME. Alergia a proteína de leche de vaca en el menor de un año. Rev Chil Pediatr. 2012; 83(1): 78-83.

12. Montero, A. Estudio descriptivo de intervencionista sobre alergia a la proteína de la leche de vaca IGE mediada en niños del área de salud de Mérida. [Tesis Doctoral]. Universidad de Extremadura: 2015.

http://dehesa.unex.es/bitstream/handle/10662/4060/TDUEX 2016 Montero_Salas.pdf?sequence=1\&isAllowed= y

13. Minchin, M. Milk matters: infant feeding and immune disorder. 1st ed. Australia; 2015.

14. Morero, A. Estudio sobre alergia alimentaria en la edad pediátrica[Tesis]. Universidad de la Coruña; 2015. http://ruc.udc.es/dspace/bitstream/handle/2183/16490/MorenoAlvarez_Ana TD 2015.pdf?sequence=2

15. Errázuriza, G., Lucerob, Y., Ceresaa, S., Gonzaleza, M., Rossela, M., Vivesa, A. Características clínicas y manejo de lactantes menores de 1 año con sospecha de alergia a proteína de leche de vaca. Rev Chil Pediatr. 2016;6(87):449-454.

16. Sánchez, E. Modelo EFQM y calidad asistencial. Rev Calidad Asistencial 2002; 17 (2): 64-66. [29] González RM. El modelo de excelencia 2000 de la EFQM y la enfermería. Gestión Hospitalaria

17. Plaza, AM. Alergia a proteínas de leche de vaca. Asociación Española de Pediatría. Protoc diagn ter pediatr. 2013; (1):51-61. Disponible en http://www.aeped.es/sites/default/files/documentos/5-aplv.pdf 


\section{Revista Electrónica Enfermeria Actual en costa Rica}

18. Bozzola M, Marchetti P, Cosentino M, Corti M, Petriz N, Parisi C. Alergia a la proteína de la leche de vaca. Evaluación de su resolución espontánea por medio de desafíos doble ciego placebo controlados. Arch de Alergia e Inmun Clín. 2015; 46 (2):44-48.

19. Brham, P., Valdés, V. Beneficios de la lactancia maternal y riesgos de no amamantar. Rev Chil Pediatr. 2017;88(1):7-14

20. Walker, WA, Shuba R. Breastmilk, microbiota and intestinal homeostasis. Pediat. Res. 2015;77(1-2):220-8. Disponible en https://www.ncbi.nlm.nih.gov/pubmed/25310762

21.Sánchez, J., Restrepo, MN., Mopan, J., Chinchilla, C., Cardona, R. Alergia a la leche y al huevo: diagnóstico, manejo e implicaciones en América Latina. Rev. Biomédica. 2014;34:143-56. http://dx.doi.org/10.7705/biomedica.v34i1.1677

22. Nieto, A., Wahn, U., Bufe, A., Eigenmann, P. et al. Allergy and asthma prevention. Pediatr Allergy Immunol 2014;25(6):516-33.

23. Denis, M., Loras-Duclaux, I., Lachaux, A. Cow's milk protein allergy through human milk. Arch Pediatr. 2012;19(3):305-12.

24. Trujillo, P. Alergia a las Proteínas de leche de vaca. Rev Cubana Pediatr. 2017; 89(3) 391-141

25. Kellams, A., Harrel, C., Omage, S., Gregory, C., Rosen-Carole, C. Academy of Breastfeeding Medicine. Protocolo clínico ABM n. ${ }^{\circ}$ 3: Tomas suplementarias en el recién nacido a término sano amamantado, revisado en 2017. Academy of Breastfeeding Medicine. 2017;12(3). DOI: 10.1089/bfm.2017.29038.aj

26. Vera, JF., Ramírez, A. Síntomas digestivos y respuesta clínica en lactantes con alergia a la proteína de leche de vaca. Rev Chil Pediatr. 2013; 84 (6): 641-649. 